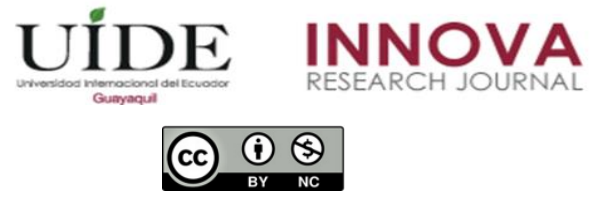

INNOVA Research Journal, ISSN 2477-9024

(Enero-Abril 2020). Vol. 5, No.1 pp. 196-205

DOI: https://doi.org/10.33890/innova.v5.n1.2020.1276

URL: http://revistas.uide.edu.ec/index.php/innova/index

Correo: innova@uide.edu.ec

\title{
Calidad de servicio en el supermercado metro de Perú
}

\section{Quality of service at metro supermarket of Peru}

Victoria Gardi Melgarejo

https://orcid.org/0000-0001-6016-8719

Carlos Oswaldo Venturo Orbegoso

https://orcid.org/0000-0002-7465-8687

Adela Jesús Faya Salas

https://orcid.org/0000-0002-7991-4133

Helga Ruth Majo Marrufo

https://orcid.org/0000-0002-5557-4269

Universidad César Vallejo, Perú

Autor para correspondencia: vicky_24_01@hotmail.com; cventuro2911@gmail.com;

lifasa1@hotmail.com; helmajo@hotmail.com

Fecha de recepción: 18 de diciembre del 2019 - Fecha de aceptación: 29 de diciembre del 2019

\section{Resumen}

El objetivo principal de este estudio fue analizar la calidad de servicio en el Supermercado Metro de Perú. El estudio se desarrolló bajo el enfoque cualitativo, la recopilación de datos se realizó a través de las entrevistas a quince clientes, con la respectiva guía de entrevista constituida por cinco preguntas alineadas a las sub categorías denominadas: tangibilidad, empatía, capacidad de respuesta, fiabilidad y seguridad. Los datos se triangularon con el software Atlas.Ti 8, el proceso implicó la codificación, la identificación de textos según los códigos, la determinación de las frecuencias, y las redes. Los resultados evidenciaron que el supermercado brinda un servicio de calidad, sustentado en la amabilidad, la asistencia, la ayuda, el conocimiento, la confianza y la familiaridad de los colaboradores con los clientes. Asimismo, dentro de las políticas de la empresa involucra a la capacitación y el reconocimiento, la sinergia de los mismos, motivan el mejor desempeño de los trabajadores, y es percibido diariamente por los clientes.

Palabras clave: calidad de servicio; fiabilidad; capacidad de respuesta; seguridad; tangibilidad

\section{Abstract}

The main objective of this study was to analyze the quality of service in the Metro Supermarket in Peru. The study was carried out under the qualitative approach, data collection was carried out through interviews with fifteen clients, with the respective interview guide consisting of five questions aligned to the subcategories called: tangibility, empathy, responsiveness, reliability, and security. The data were triangulated with the Atlas. Ti 8 software, the process involved coding, text identification according to codes, frequency determination, and networks. The results showed that the supermarket provides a quality service based on the friendliness, assistance, help, knowledge, trust, and familiarity of employees with customers. Likewise, within 
the company's policies, it involves training and recognition, their synergy, motivates the best performance of workers, and is perceived daily by customers.

Key words: quality of service; reliability; responsiveness; security; tangibility

\section{Introducción}

En una sociedad postindustrial la calidad de servicio constituye una de las prioridades en la industria de los supermercados, porque el cliente es cada vez más exigente para realizar su compra. Las empresas de retail diseñan sus procesos para ofrecer un servicio de calidad, razón por la cual constantemente realizan cambios en las góndolas, distribución, campañas publicitarias agresivas, trabajan bajo la estrategia de costos con el único objetivo que el cliente reciba un mejor servicio y se sienta satisfecho, el mismo que se reflejará en las ventas y rentabilidad.

Este proceso de cambio económico, tecnológico, político y social está inquietando a todas las organizaciones empresariales que buscan extender sus activos mediante la satisfacción del cliente, dentro de este tenor las empresas de retail vienen alcanzando un revelador incremento. Se ha evidenciado que en estos negocios resulta ser insuficiente a nivel mundial la calidad de atención (Rubio, 2014).

Carrizo, Freitas y Ferreira (2017), Bernal, Pedraza, Lavín y Monforte (2017), Ibarra, Puerto y Suárez (2018) determinan una relación objetiva y significativa entre la calidad de servicio y la satisfacción de los usuarios, con la finalidad de conseguir la lealtad del cliente, razón por la cual deben gestionar experiencias con la marca, la calidad de la atención, la complacencia y la confianza.

Quijano, Rojas, Robayo (2018), Vanegas, Restrepo, Barros, Agudelo (2018), Vargas y Cadena (2017) mencionan que, con el fin de constituir un innovador plan de mejora continua, se debe aprovechar las debilidades, y fortalecer escenarios que buscan la satisfacción del usuario.

Así mismo Cruz, López y Ruiz (2016), Duque, Saldarriaga y Bohórquez (2017) reconocen que el perfeccionamiento de la calidad impacta en la mejora continua de las organizaciones, refrendando el éxito de un sistema sostenible de gestión de calidad. Cabe precisar que la calidad se admite como el cumplimiento de las exigencias, donde se comprueba que, el producto y el servicio sean capaces de satisfacer los requisitos de los usuarios, además de entender sus necesidades y lograr clientes fieles.

En el caso peruano, Ugarte (2019) sostuvo que la calidad de servicio implica certificar que cada individuo reciba el servicio más conveniente según su solicitud y logre satisfacer sus necesidades. Mendoza (2015) refieren que los clientes no solo deciden comprar a una cierta marca por la calidad, sino también porque se logra mejorar las relaciones entre el comprador y vendedor.

A partir de ello nace la necesidad de ser eficaces en el servicio para incrementar la satisfacción de los compradores y clientes en las empresas retail. Razón por la cual las empresas deben de realizar capacitaciones al personal de tienda, evaluación constante al capital humano, 
proyectar el presupuesto en base a los requerimientos de los consumidores, formación sobre políticas de asistencia, planear y organizar la cobertura de cajas de atención y una revisión constante de equipos.

León, Ríos y Salazar (2016) establecieron que la implementación de un plan de mejora en la gestión de servicios agrega valor al segmento, beneficiando al cambio de imagen y avance comercial, además generan mayor confianza y preferencia en los compradores. Ventura y Mamani (2017) manifiestan que, implementando las tres maniobras primordiales que son: a) la exclusividad en los productos; b) atención diferenciada en sus servicios; c) fortalecimiento en la atención al cliente; se logra obtener ventaja competitiva.

Por lo tanto, la calidad de servicio se apoya en la teoría Humanista de Maslow y Rogers (1954) quienes revelaron que la capacidad humana y su progreso son el foco del desarrollo humano. La teoría al desarrollarse en distintos campos, primordialmente en empresas del siglo XXI se desarrolla como un instrumento de estudio para comprender a la personalidad, que beneficia la salud mental y la evolución personal en el trabajo, con la familia y el entorno que nos rodea. Bertalanffy (1968) y Bertoglio (1994) refieren que la teoría de sistemas empleada debidamente ayuda a incrementar la producción de las organizaciones, a generar más activos, a optimizar su dirección y satisfacer a los requerimientos de los clientes.

Por ende, los enfoques serán útiles en departamentos y situaciones específicas, sin perder el contexto del problema, las variables que intervienen y la relación que preexisten; para hallar la salida a las dificultades que se presenten (Tamayo, 1999).

Barroso (2000), define que la calidad de servicio es aquello que el cliente espera encontrar y recibir, es la conformidad de la atención al momento que eligen y hacen su compra. Calidad es buscar permanente la perfección en la producción, en la interrelación cliente y vendedor. La calidad de servicio es una apreciación muy difícil de manifestar e impreciso de medir.

Parasuraman, Zeithmal y Berry (1985) y Drucker (1992) argumentaron que la calidad es la valoración que el comprador plasma por lo bueno y excelente del producto o servicio, es una respuesta a la conformidad o disconformidad con relación a lo esperado o lo deseado, conforme a las expectativas de los usuarios. Del mismo modo Ishikawa (1988) y Crosby (1996) sostuvieron que es todo aquello que se desarrolla en el proceso de cambio entre el cliente y los trabajadores, cabe precisar que no es solo el producto, es la calidad manifestada de forma precisa; es crear, producir, conservar un producto que resulte alcanzable a los consumidores, además de una asistencia post venta, efectiva y eficiente.

El servicio es una diligencia o asistencia que una parte ofrece a otra, es intangible, se inicia al interactuar el usuario con los trabajadores (Gronroos, 2007; Kotler y Bloom, 1984). Albrecht (2017) refiere que es un acumulado de técnicas y programas encaminados al servicio del cliente. Son conductas realizadas por el capital humano, tienen un valor económico, por lo que se esperan beneficios o satisfacción como un factor de diferenciación. Vértice (2011) arguye se refiere al conjunto de prestaciones que los clientes esperan del producto, además de la imagen y la notoriedad de este. 
La medición de la calidad de servicio es resultado de la importancia dada a la misma, se acepta en general que, si se facilita un buen servicio, se consigue mayores beneficios económicos. El modelo SERVQUAL propuesto por Parasuraman, Berry, Zeithaml (1988) está constituido por cinco dimensiones: a) fiabilidad: se refiere a la habilidad para dar el servicio prometido de forma íntegra y esmerada, es decir, que la organización cumple con sus promesas sobre entregas, suministros del servicio, solución de problemas y fijación de precios; (b) capacidad de respuesta: es la disposición para auxiliar a los clientes y para prestarles una asistencia rápida y apropiado, se refiere a la atención eficaz al atender las solicitudes, responder inquietudes y descontentos de los clientes y dar solución a sus inconvenientes; (c) seguridad: es la prudencia y atención de los empleados y sus destrezas para inspirar credibilidad y cordialidad; (d) empatía: se refiere al nivel de atención personalizada que ofrecen las compañías a sus usuarios, el cual se debe transmitir por medio de un servicio personificado o adecuada al gusto del consumidor; y (e) elementos tangibles: son los aspectos de la infraestructura física, es decir las instalaciones, como la construcción, equipos y materiales.

\section{Método}

El enfoque de la investigación fue cualitativo, se enfoca en comprender y ahondar en los fenómenos, examinándolos desde la perspectiva de los colaboradores en su ambiente originario y la relación con el contexto (Hernández, Fernández y Baptista, 2014), asimismo hace referencia a los atributos, a la particularidad que se percibe, a la narración, al relato y al comportamiento del sujeto (Naupas, Valdivia, Palacios y Romeo, 2018).

La recolección de datos se realizó en su contexto original y habitual de los participantes o elementos de observación. Rojas (1997) mencionó que el volumen y el tipo de datos cualitativos que se logren en el trabajo de campo, deberán estar completamente justificados por los objetivos del estudio, en vista que se corre el riesgo de seleccionar testimonios de poca o ningún provecho para realizar un análisis correcto del problema.

La técnica aplicada fue la entrevista a catorce clientes voluntarios, la comunicación con los participantes se llevó acabo sobre la base de la guía que está constituida con las siguientes peguntas a) ¿Cómo percibió la actitud del colaborador y el empleado en su experiencia de compra en Supermercado Metro? ¿Por qué? b) ¿En su opinión, el Supermercado Metro ofrece productos saludables y satisfacen sus expectativas?, ¿Por qué? c) ¿En su opinión, los trabajadores del Supermercado Metro lo ayudan cuando usted llega al establecimiento? ¿Por qué? d) ¿En su opinión los trabajadores del Supermercado Metro se esfuerzan por satisfacer sus necesidades?, ¿Por qué? e) ¿En su opinión las instalaciones físicas y los equipos tecnológicos de Supermercado Metro son adecuados?, ¿Por qué?

El método de análisis que se utilizó fue la triangulación, permitió confirmar los resultados y efectuar la validación de testimonios cualitativos. En la actualidad se han desarrollado programas como el Atlas.ti que nos permitió dividir y codificar los datos, se ejecutó los conteos, además de visualizar la relación entre el investigador y las unidades de estudio, las categorías, los temas y los documentos primarios (Hernández, Fernández y Baptista, 2015). 
En este contexto la categoría calidad de servicio se desglosa en sub categorías como: a) Fiabilidad, con sus indicadores eficiencia y eficacia; b) Capacidad de respuesta, con sus indicadores ayuda y servicio; c) Empatía, con atención personalizada e interés por el cliente; d) Seguridad, con sus indicadores conocimiento y confianza; y e) Tangibilidad, constituida por las instalaciones físicas, equipos tecnológicos, presentación del personal y materiales de comunicación, como observamos en la figura 1.

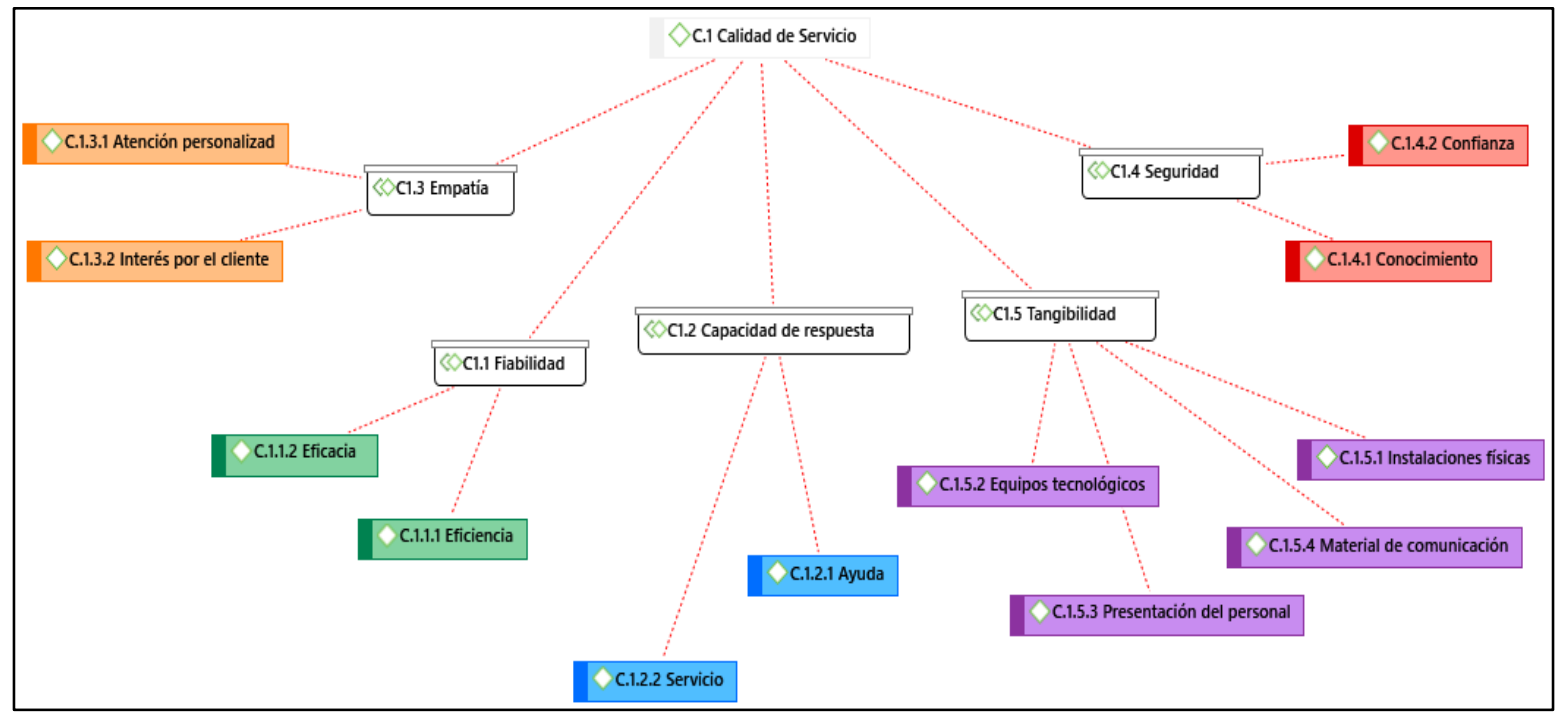

Figura 1. Red de la Calidad de servicios con sus sub categorías e indicadores

\section{Resultados}

Los clientes consideran que la fiabilidad a través de su indicador eficiencia es el principal elemento que mide la calidad del servicio del Supermercado Metro de Breña, porque mide la aptitud, la disposición, habilidad, soltura, destreza y practica de sus colaboradores al momento de la interacción cliente y trabajador, que no es más que el producto de la eficacia, es decir del vigor, energía y virtud que demuestran los colaboradores en la atención.

La empatía con sus indicadores atención personalizada e interés por el cliente, ejercen también inciden drásticamente en la calidad de servicio como lo expresan los usuarios, porque necesitan que comprendan sus necesidades y deseos, intentando experimentar de forma objetiva la necesidad del cliente, es decir demostrar la consideración, preocupación y la capacidad de ayuda que requieren los compradores.

Con respecto a la capacidad de respuesta con sus indicadores ayuda y servicio a los clientes, consideran que la asistencia, el favor, el auxilio que los trabajadores brindan desde el momento que ingresan a las instalaciones, resultan ser importantes para determinar su satisfacción, sienten que son importantes para los colaboradores, así como para el establecimiento.

En cuanto a la seguridad, los clientes perciben confianza respecto a los productos que consumen y la información que reciben, demostrando conocimiento, a la vez preparación para lograr satisfacer los deseos y necesidades de los consumidores. 
En referencia a la tangibilidad, los clientes también manifiestan que las instalaciones físicas juegan un papel importante para la distribución adecuada de los productos, y facilita la rápida ubicación de los departamentos, los equipos tecnológicos y las máquinas registradoras que facilitan la efectividad y rapidez en la atención. También es súper importante la presentación del personal, la misma que aporta a la imagen del establecimiento, tal como se expresa en la figura 2.

Los entrevistados manifestaron sentirse conforme con el trabajo de los colaboradores, porque son competentes, conocen sus funciones y están informados del manejo y el movimiento de la empresa. También manifiestan que son amables, están siempre predispuestos a brindar ayuda, razón por la cual expresan ante las inquietudes respuestas rápidas.

Asimismo, los colaboradores muestran respeto al cliente, que se traduce como aprecio, atención y consideración, como parte de la política de la empresa, utilizan expresiones adecuadas, son claros y precisos, utilizan términos y palabras que los usuarios entienden, escuchan con atención los deseos, las dudas, en resumen, atienden las necesidades, y demuestran interés por el usuario.

A esto se suma la confianza, los participantes afirmaron que la calidad del servicio depende especialmente del buen trato, es decir que demuestren cortesía, amabilidad y agrado del producto por sus atributos: costo, variedad y la calidad.

De esta manera, esperan que la distribución de los productos y letreros les permita la ubicación rápida de los productos y la variedad de los mismos, además de ser consciente del costo. También mencionaron que el supermercado aplica toda clase de estrategias, los colaboradores se muestran carismáticos y hasta "graciosos", logrando con dicho comportamiento cubrir las expectativas de los clientes, capacidad de respuesta y satisfacción, al mismo tiempo hacen que el proceso de espera ante alguna consulta o reclamo sea el más corto posible.

Del mismo modo en la relación de la sub categorías capacidad de respuesta con la empatía, los entrevistados manifestaron que, el interés por el cliente de parte de los trabajadores determina su satisfacción, es decir los clientes sienten ser importantes y valorados por la empresa.

Además, se preocupan por los detalles, la variedad de los productos, las diferentes presentaciones y disponibilidad de los mismos. También aprecian recibir una atención personalizada que la resumen en trato directo y personal, un correcto servicio prometido de forma integral y honrada, además de atender de manera especial sus gustos y preferencias, su apreciación y deleite por las tendencias de moda, los descuentos y las promociones.

También refieren que los colaboradores practican la empatía, en alguna situación se vieron con el problema de pago o devolución del producto, razón por la cual los colaboradores brindaron atención y apoyo oportuno para solucionar dicho inconveniente, el mismo que mermo el tiempo y disgusto, en resumen, la solución rápida es valorada. 


\section{Conclusiones}

La calidad de servicio que brinda el supermercado es eficaz, los colaboradores brindan una atención personalizada, demuestran interés por el cliente, denotan predisposición para ayudar, brindar seguridad y confianza, las instalaciones físicas están bien distribuidas y los equipos tecnológicos que utilizan ayudan a lograr la satisfacción y cubrir las expectativas de los clientes.

El interés por el cliente y la atención personalizada determina el estado de satisfacción del cliente, básicamente por la ayuda, la asistencia y el servicio recibido. El conocimiento, la intuición, la confianza y la familiaridad de los colaboradores con los clientes genera fiabilidad y seguridad para que se reitere la compra y familiaridad con el establecimiento, siendo considerado como prioridad para futuras operaciones comerciales. 


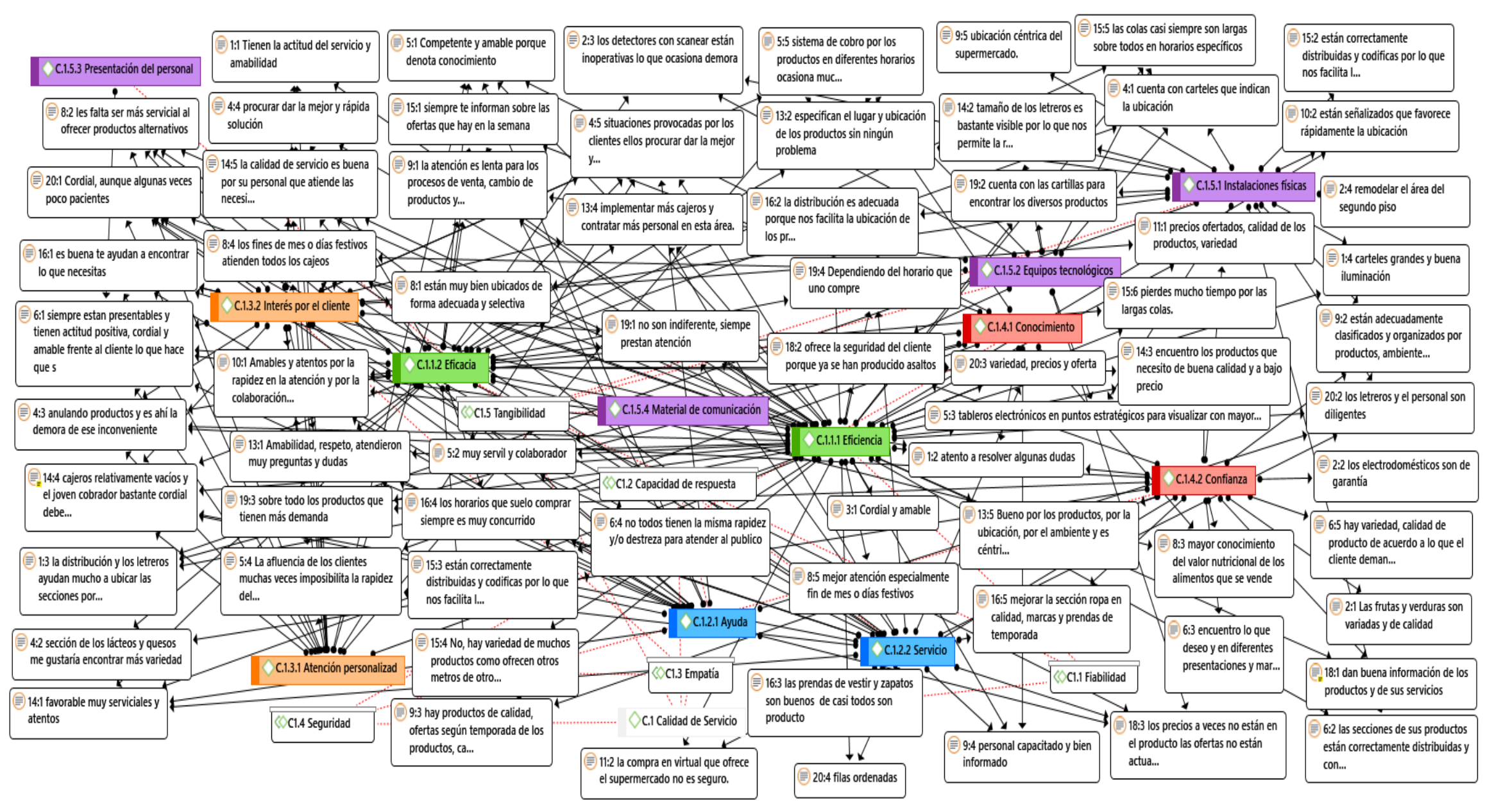

Figura2. Red de la Calidad de servicio en un supermercado de Lima 


\section{Bibliografía}

Albrech, K. (2017). Como Negociar con Exito. Ediciones Juan Granica-Colección Managment.

Barroso, C. (2000). Factores organizativos que influyen en las percepciones de los clientes en el ambito de los servicios. Conecuencias para la rentabilidad. Proyectos de investigación. Sevilla.

Bernal, I., Pedraza, N., Lavín, J., \& Monforte, G. (2017). Service Quality and Users’ satisfaction assessment in the health context in Mexico. Journal Management, 37-46. doi:10.25100/cedea.v33i574510

Bertalanffy Ludwin, V. (1968). General System. Theory Fundation Develoment Aplications. New York Brasiller.

Bertoglio, O. (1994). Introducción a la Teoria General de Sistemas. Noriega: Limusa Editores.

Carrizo, A., Freitas, P., \& Ferreira, V. (2017). The effects of Brand Experiences on Quality satisfaction and Loyalty: An empirical Study in the Telecommunications Multiple-play Service Market. Innovar Journal, 23-36.

Crosby, P. (1996). Hablemos de calidad. Mexico: McGraw-Hill Interamericana S.A.

Cruz, F., Lopez, A., \& Ruiz, C. (2017). Sistema de Gestion ISO 9001-2015 Tecnicas y Herramientas de Ingenieria de Calidad Para su Implementación. Ingenieria, Investigación y Desarrollo, 59-69.

Drucker, P. F. (1992). La Innovación y el Empresario Innovador (Vols. 978-84-350-1428-1). Edhasa.

Duque , G., Saldarriaga , F., \& Bohorquez, E. (2016). El servicio al cliente y sus problemáticas actuales: tendencias investigativas. Criterio, 395-408.

Gronroos. (2007). Marketing y Gestion de Servicios: La gestion de los momentos de la verdad y la competenccia en los servicios. España: Diaz Santos.

Hernández , R., Fernández, C., \& Baptista, M. (2014). Metodología de la investigación 6ta Edic. Mexico: Mc Graw Hill Educación.

Hernández Sampieri , R., Fernandez Collado, C., \& Baptista Lucio, P. (2015). Metodológia de la Investigación (Vols. 968-422-931-3). MEXICO: McGraw-Hill Interamericana.

Ibarra, K., Puerto , G., \& Suarez, C. (2018). Quality of service evaluation based on network slicing for sofware defined $5 \mathrm{G}$ system. Tecnológicas, 27-41.

Ishikawa, K. (1988). Practica de los Circulos de Control de Calidad. Tecnologias de Gerencia y producción.

Klotler, P., \& Bloom, P. (1984). Marketing Professional Service. N.J Prentice Hall: Englewood Cliff.

León , G., Rios, A., \& Salazar, B. (2016). "Modelo de gestión de servicio para el desarrollo de mercados tradicionales". Tesis de la Universidad Peruana de Ciencias Aplicadas.

Maslow , A., \& Rogers , C. (1954). Introducción a la teoria Humanista. Noriega Limusa Editores.

Mendoza, C. (2015). "Relación entre la calidad de servicio y la satisfacción del cliente en el Supermercado Metro Chilca - Huancayo". Tesis de la Universidad Nacional del Centro del Perú.

Ñaupas Paitan, H., Valdivia Dueñas, M., Pañacios Vilela, J., \& Romero Delgado, H. (2018). Metodología de la Investigación Cuantitativa-Cualitativa y Redacción de Tesis (Vol. 9789587628760). Ediciones de la U.

Esta obra se comparte bajo la licencia Creative Common Atribución-No Comercial 4.0 International (CC BY-NC 4.0) 
Parasuraman, A., Zeithaml, V., \& Berry , L. (1985). Calidad Total en la Gestion de ServiciosCómo lograr el equilibrio entre las percepciones y las expectativas de los consumidores. Madrid-España: Diaz de Santos.

Parasuraman, A., Ziethaml, V., \& Berry, L. (1988). SERVQUAL: a multiple item sacale fo measuring consumer perceptions of service quality. Jorunal of Retailing, 12-40.

Quijano, J., Rojas, S., \& Robayo, O. (2018). Service quality for Continuing education in online learning enviroments. Administración, 22-34.

Rojas Soriano, R. (1997). Métodos para la investigación social-Una Proprosición Dialectica. Mexico: Plaza y Valdez.

Rubio , G. (2014). La Calidad del servicio al cliente en los grandes supermercados de Ibague: un análisis desde la escala multidimensional (SERVQUAL). Colciencias, 54-64.

Tamayo, A. (1999). Teoria General de Sistemas. Depto. de Ciencia, 84-89.

Ugarte, O. (2019). Gobernanza y Rectorïa de la Calidad en los Servicios de Salud en el Perú. Peru Med Exp Salud Pública. doi:http//dx.doi.org/10.17843/rpmesp2019.362.4495

Vanegas, J., Restrepo, J., Barros, G., \& Agudello, G. (2018). Service quality in Medellin hotel using peceptual maps. Journal Management, 40-45. doi:10.25100/cdea.v34i60.5927

Vargas, M., \& Cadena , E. (2017). Review of Quality of Service (QoS) mechanics over IP Multimedia Subsystem (IMS). Ingenieria y Desarrollo, 1-20. doi:10.14482/inde.35.1.8952

Ventura, I., \& Mamani , L. (2017). "Influencia de la cultura de calidad del servicio en la satisfacción del cliente de Supermercado Plaza Vea del Distrito de Independencia, Lima". Tesis De la Universidad Inca Garcilazo de la Vega.

Vertice, L. (2011). Tenicas de Información y Atención al Cliente Consumidor. Fundción Emprende. 